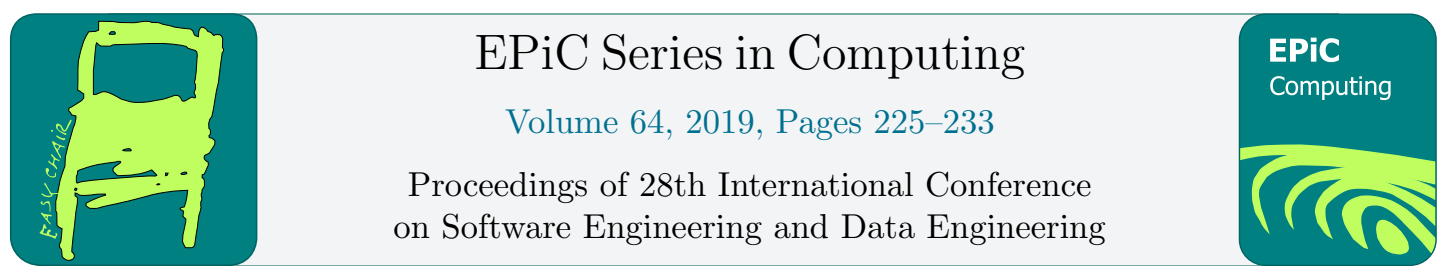

\title{
Quack: A Youth-Targeted Game for English Comprehension
}

\author{
Alexander Redei \\ Department of Computer Science \\ Central Michigan University \\ Mt. Pleasant, Michigan, U.S.A. 48858 \\ redeila@cmich.edu
}

\begin{abstract}
Second grade is a critical year in the development of a child's understanding of literature. Studies have shown that students who struggle with reading at the second grade will continue to struggle with reading for the rest of their lives.

The purpose of this paper is to introduce a new method for increasing English comprehension for at-risk youth. In this paper we propose a fun and easy-to-use game called Quack. Our approach is to leverage new technologies with an existing school program, such as one-on-one mentoring, in addition to a targeted English comprehension game to enhance the educational experience of second graders struggling with reading comprehension. The Quack game implements a spelling challenge system to test the student's vocabulary and spelling ability. Part of the game, an options system, allows instructors and students to customize the experience to each individual's needs. Quack provides a novel approach to educational gaming through three new concepts: (i) Quack is free to use and open source (ii) Quack is customizable to the individual's English comprehension needs (iii) Quack incorporates a rewarding English-comprehension system, effectively "gamifying" learning proper spelling.
\end{abstract}

\section{Introduction}

The aim of the Quack game is to provide a fresh new way for youngsters to get involved in reading [1]. The motivation for this software comes from volunteering with second grade students in the local elementary school and observing their struggle with English comprehension.

References [2], [3], [4], and [5] discuss the consumer appeal of using high resolution graphics in a game. In addition, another conclusion that can be drawn from the background research is that "gamified" methods of learning are becoming more prevalent and have been highly successful.

Quack has adopted this approach, and utilizes DirectX 11 graphics to render the scenes, GUI, and game objects in Quack.

F. Harris, S. Dascalu, S. Sharma and R. Wu (eds.), SEDE 2019 (EPiC Series in Computing, vol. 64), pp. $225-233$ 
A new trend that is emerging is called "edutainment" (essentially, educational and entertainment content in one). In summary, the edutainment business is a hot industry and has been highly successful as evidenced by Neilson Entertainment reporting a 25\% increase in their edutainment sales from 2007 $[6,7]$. Another example, Dora the Explorer [8], pulled in \$250MM in sales in the last four years.

The remainder of this paper is structured as follows: Section I describes the inadequacy of current commercial software; Section II, is focused on the solution, and proposes our alternative approach. Section III presents a product comparison between Quack and two main related works. Section IV shows what has been done with the prototype of Quack, including several screenshots of it in action. Finally, Section V ends the paper with a conclusion and future work.

\section{Problem}

The goal of Quack is to duplicate the successful "edutainment" strategy that has been proven in television, and apply it to an English comprehension video game. Our vision is to offer schools an educational tool (in the form of a spelling challenge game) that they can use to improve 2nd grade level reading comprehension. Second grade is an especially important year for reading comprehension, and that is why our game targets specifically that grade-level. The game will be used in conjunction with existing teaching structures to provide a positive, rewarding environment for children to explore spelling.

One of the best ways to improve one's spelling ability is to read lots of content [9]. The body of research suggests that encountering new words frequently helps with retention, and with understanding the way the word is spelled. In fact, it is one of the techniques that has been shown to advance SAT scores. On this principle, programs have been developed to target at-risk youth and provide them with tutoring/mentoring on reading. Most programs involve pairing the child with a volunteer mentor, who will read books to them, or play word games with them. These programs have been shown to have a positive impact on the child's development, but rarely will the children continue to challenge themselves with reading at home, after the mentoring session is over.

This presents an opportunity for a new piece of software to deliver interaction that extends beyond the classroom.

\section{Solution}

Quack aims to provide an edutainment game targeted to at-risk 2nd graders to encourage them to read and interact with English words outside the classroom. We present a simple state-diagram of the program and installation flow of Quack using Figure 1.

Quack is designed using an n-tier approach, with presentation, business, and utility layers included. There are eight main classes that handle all the processing for Quack. Since each class lives in its own thread, all exception handling is being done through the thread manager. In addition, all input and output, are being dealt with by the thread manager and the GUI driver, as shown in Figure 2. 


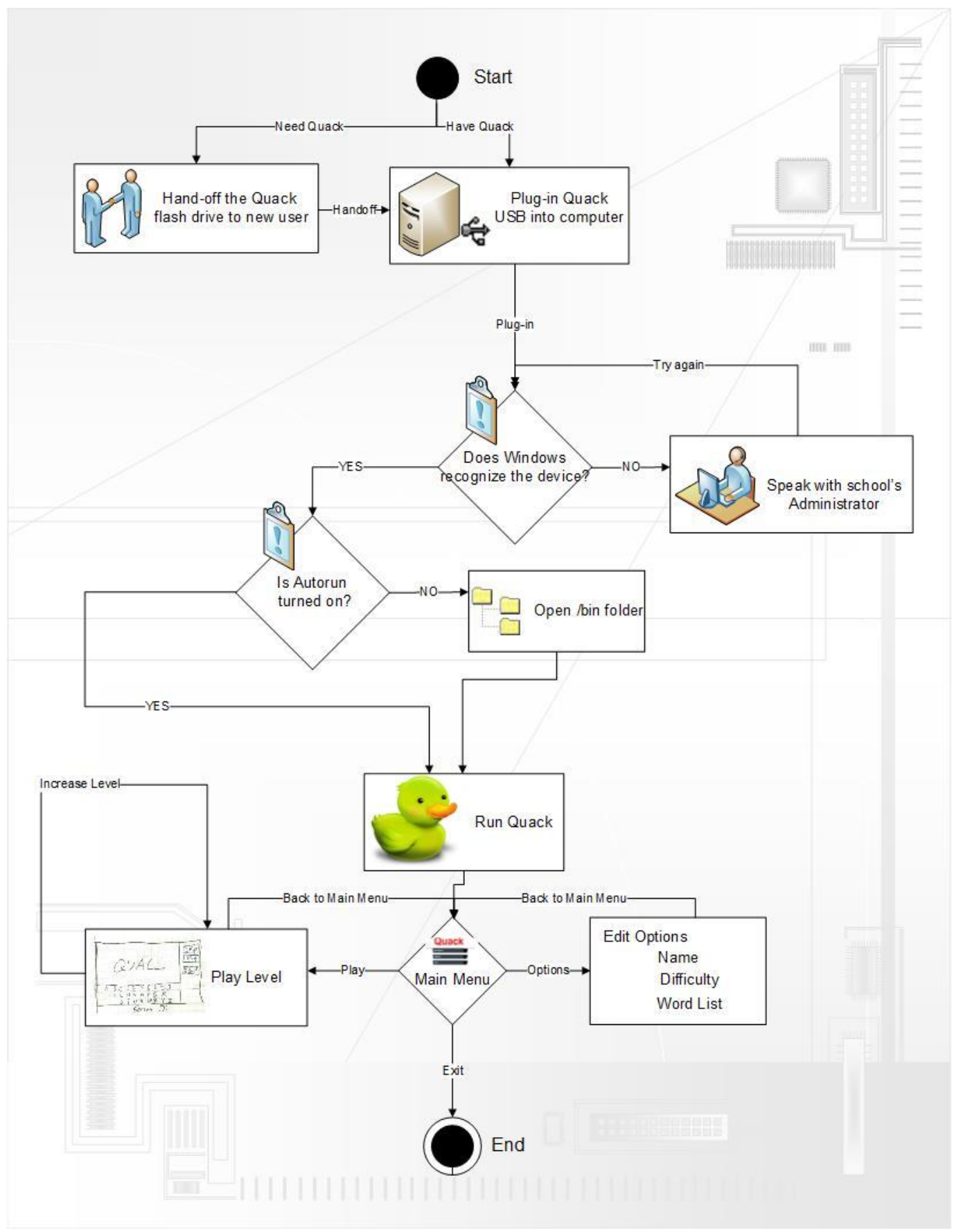

Figure 1: A state-diagram of the Quack program 


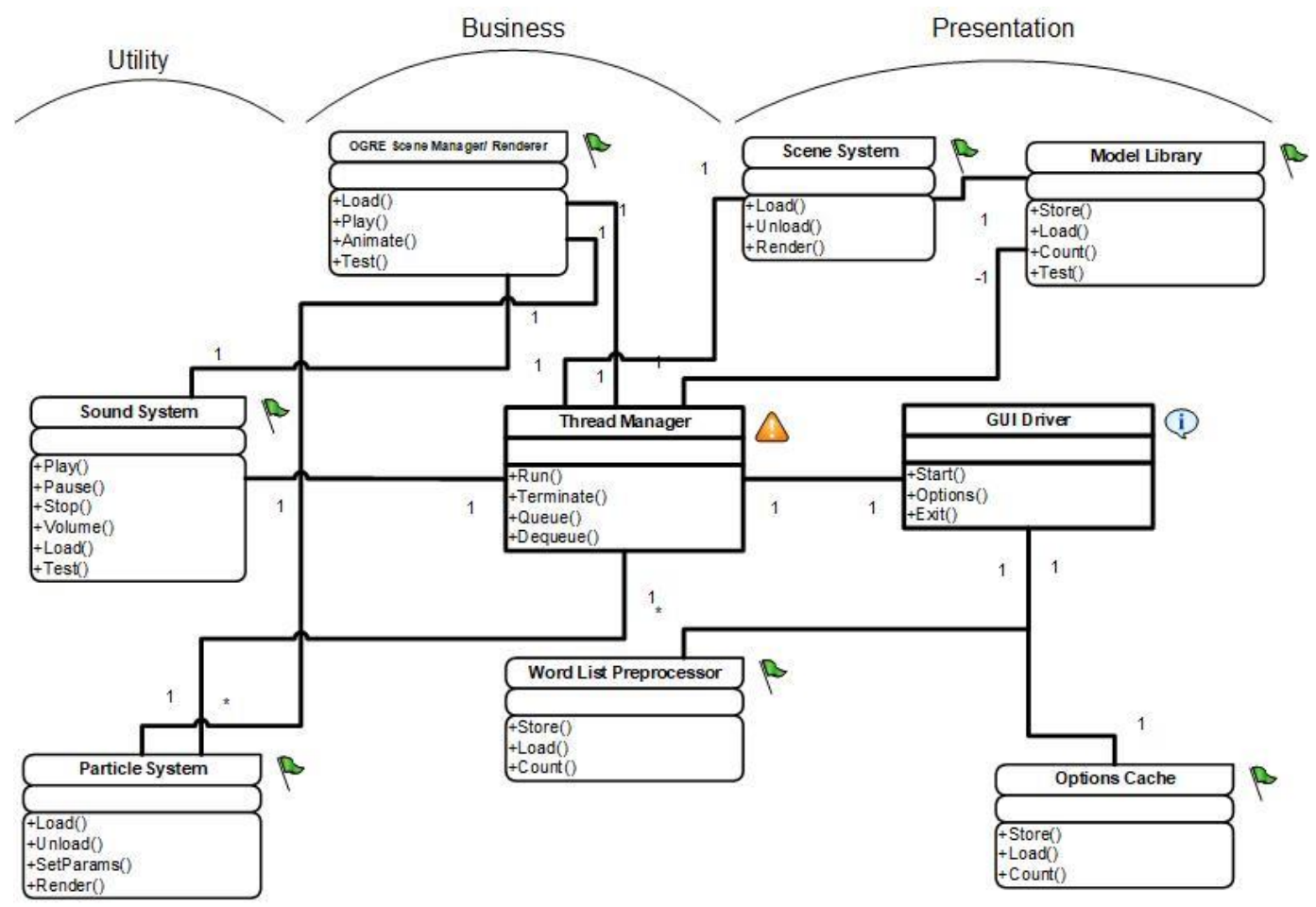

Figure 2: System Level Diagram. The orange triangles represent critical components, the informational bubbles represent components the user will interact directly with, and the green flags represent system classes.

\section{Comparison with Related Works}

There are two commercial products that in essence are similar to the work we have done in Quack: Reading Rabbit and the Dr. Seuss Learning System [12]. Quack distinguishes from them by offering three product differentiators not available, to the best of our knowledge, in other English comprehension software. If we provide a superior product, we hope that Quack will gain recognition and be the software of choice for an English game that emphasizes language learning.

The first product differentiator is that Quack does not require any installation on the host machine. Quack is built as a self-contained portable app so it can run off a flash drive, which enables high portability of the software. We are not aware of other English comprehension games at this time (other than Web perhaps) which provides this level of portability.

The second thing that makes the prototype of Quack unique is that utilizes modern graphics and audio technology. Table 1 lists out the technological advantages of Quack in comparison to similar products.

The third distinguishing characteristic of Quack is that the software is being offered on the internet for free, and is available as open-source software through the GPL license. 
Table 1: Quack Technology Comparison

\begin{tabular}{lrrr}
\hline Feature & Quack & Dr. S & RR \\
\hline Max Resolution & $2048 \times 1920+$ & $800 \times 600$ & $800 \times 600$ \\
Texture Resolution & $1048 \times 1024$ & $215 \times 215$ & $215 \times 215$ \\
Colors Supported & 32 million & 256 & $16 \mathrm{k}$ \\
Stand-alone & YES & NO & NO \\
3D graphics & YES & NO & NO \\
Audio Quality & $94 \mathrm{kHz}$ & $16 \mathrm{kHz}$ & System Beeps \\
\hline
\end{tabular}

\section{Results}

Thus far, we have identified the key audience for our prototype English comprehension software, Quack, and detailed the need for this particular group (2nd graders with at-risk comprehension scores) to spend more time reading and interacting with word-play so they do not have difficulties with English in the future. 2.

Currently, Quack has a working prototype. For a full account of the progress made so far, see Table

Table 2: Progress Made on Quack Project

\begin{tabular}{lrr}
\hline Date & Item & Status \\
\hline $3 / 6$ & Concept & $\square$ \\
$3 / 27$ & Specification & $\square$ \\
$4 / 20$ & Design & $\square$ \\
$9 / 23$ & Development / Integration & $\square$ \\
$10 / 5$ & Initial Quack Demo & $\square$ \\
\hline
\end{tabular}

Each screen in Quack has limited controls to the interface to simplify the interaction. Figures 3-6 show off, respectively, Quack's main menu, options, and configurability.

Specifically, in Figure 3 below the user is presented with choices such as changing their displayed name, which is shown in greater detail in Figure 4, changing the difficulty of the game, or incorporating a custom word list, as shown in Figure 5. The work list in this screen is used to formulate the spelling challenges in the game. The source code for quack can be downloaded at http://github.com/asmalex/Quack/

\section{Conclusions and Future Work}

As a future enhancement, we plan to add better reporting functionality in the form of tracking scores and allowing passive users to access scores across the network. For example, the teacher could access the school network to view a summary of all their children's progress in the game. In addition, the school principal could use the network to connect to a local quack server and gauge progress in the game for the whole school. 


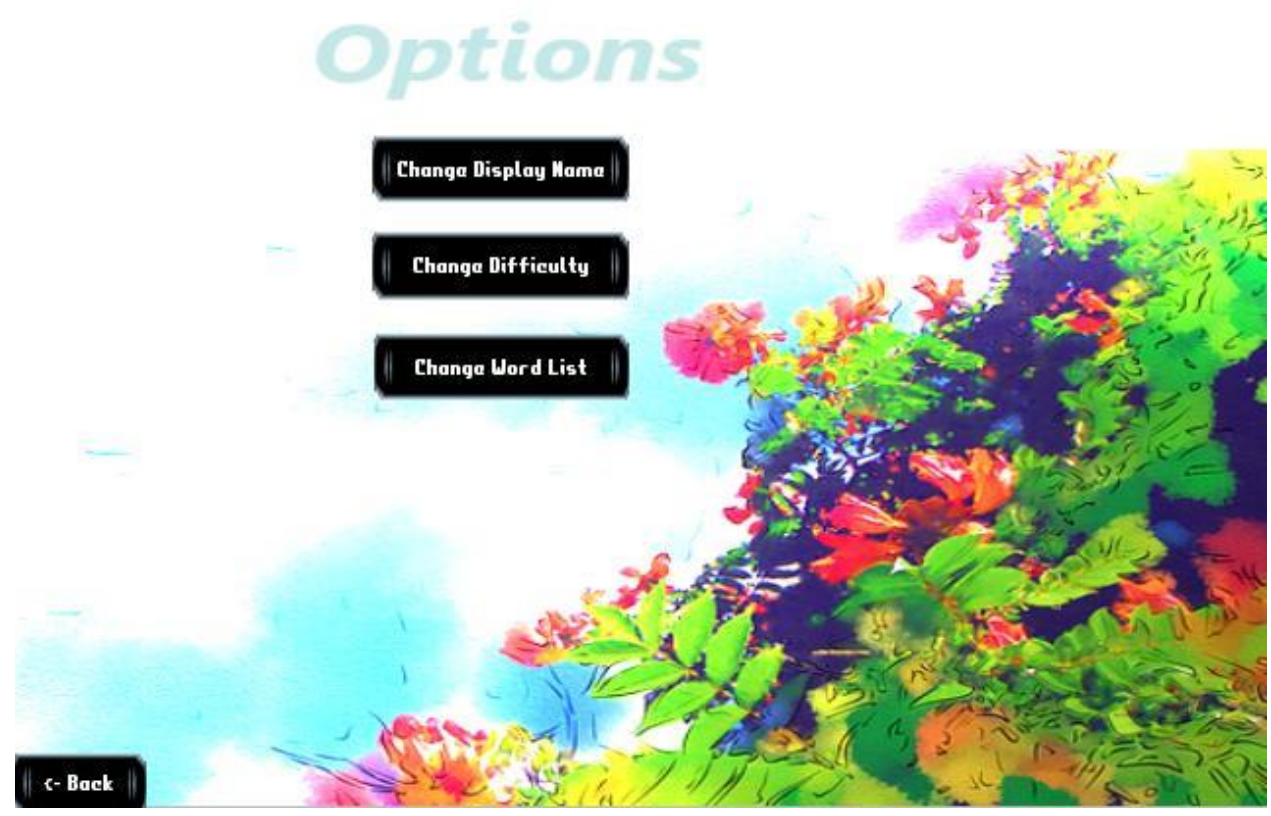

Figure 3: Quack Options Screen

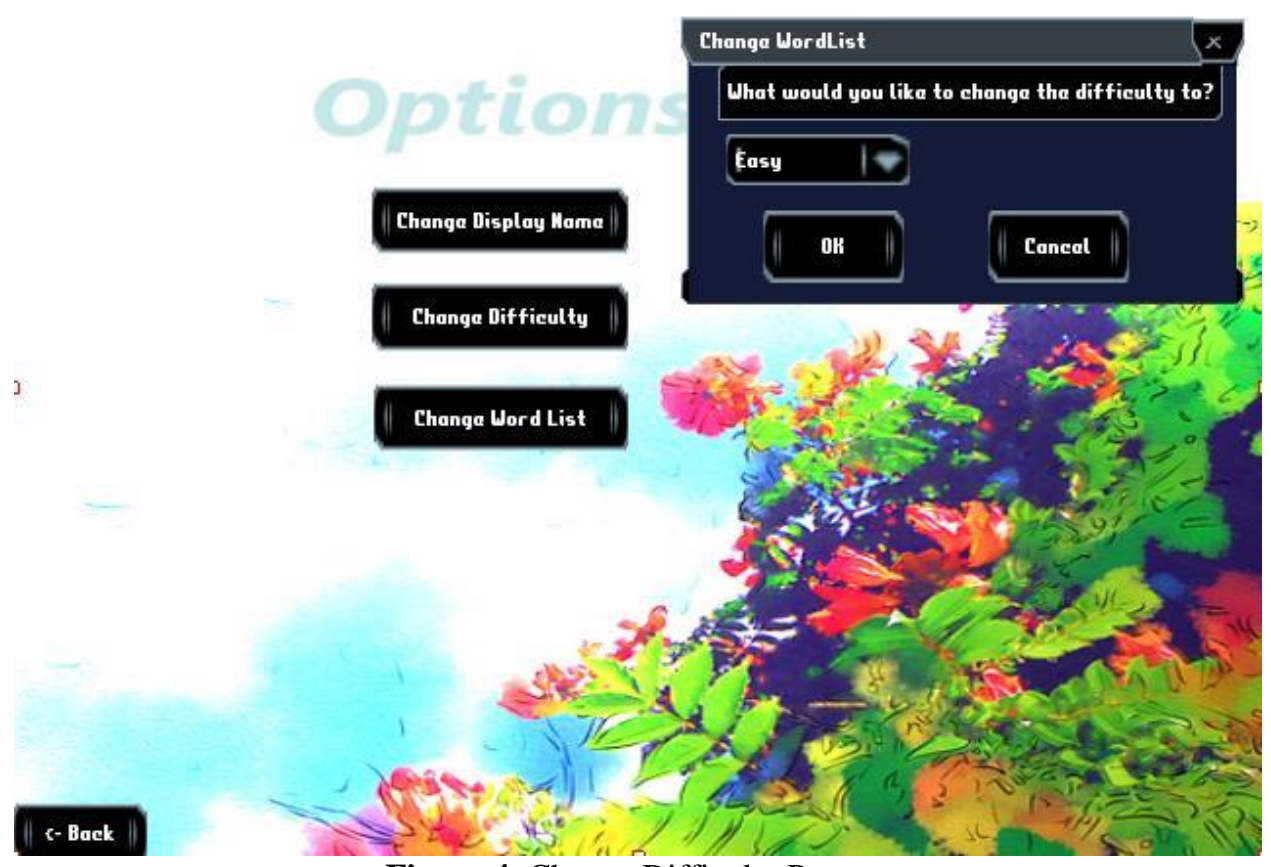

Figure 4: Change Difficulty Prompt 


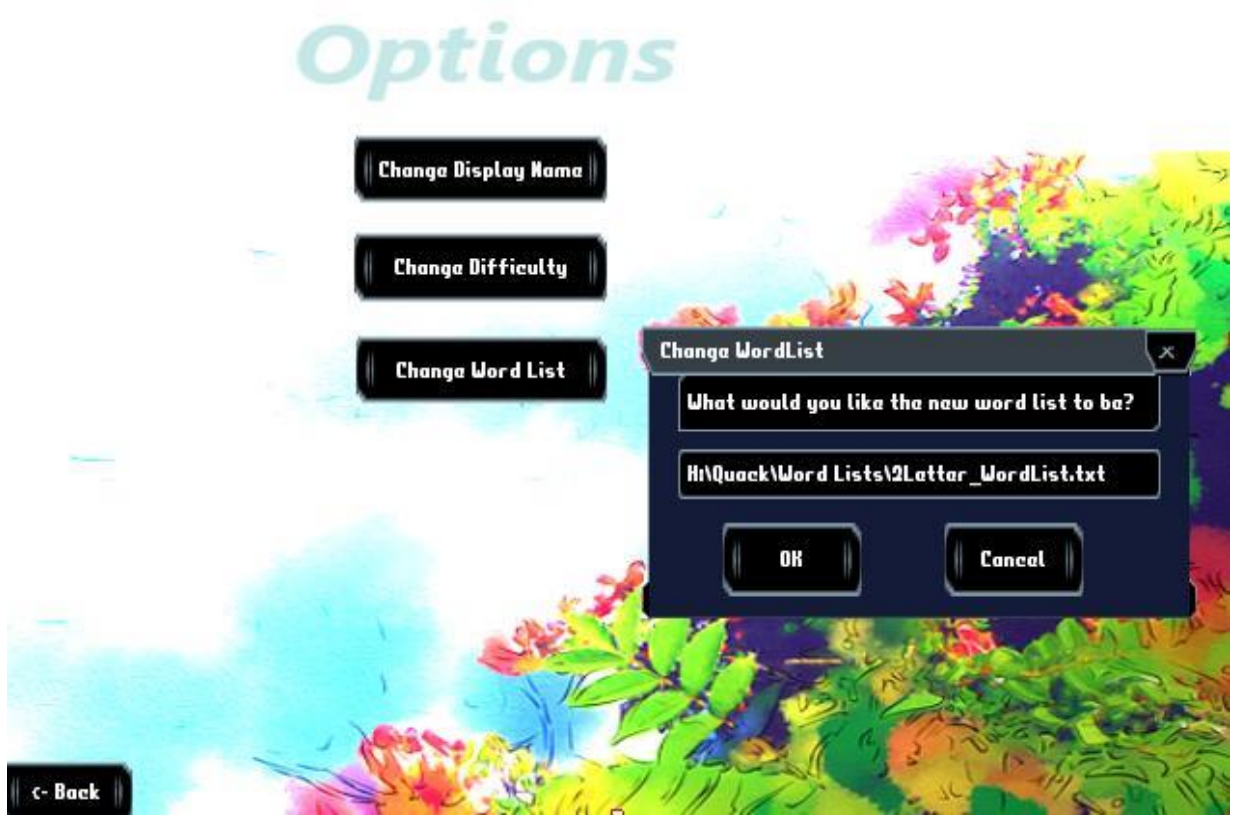

Figure 5: Change Word List Prompt

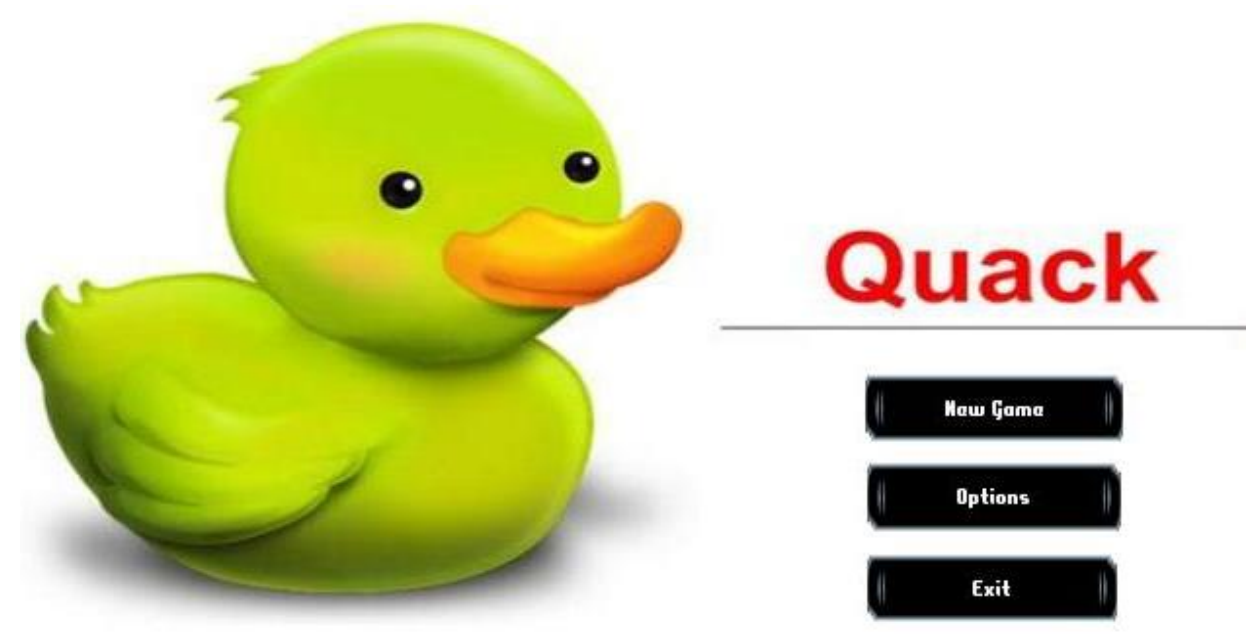

Figure 6: Quack Main Menu 
Another future enhancement we would like to provide for Quack is the ability to get or play the game online. We would like to leverage the internet to help distribute Quack to schools, or allow a parent to download an offline copy of the game for free.

The goal is to host a Quack distribution server which will provide a website for potential users to learn about Quack, and a free way for parents, schools, or children to download the game. In the future we'd like to develop a comprehensive validation / evaluation rubric to better gauge student learning outcomes.

Finally, we would like to eventually expand support for Quack beyond PC and Microsoft Windows.

Particularly, we plan to target a mobile platform to allow users to take Quack with them wherever they go. A child could use a cell phone to download and play the game on their cell phone.

Figure 7 shows a diagram of what these three features might look like in a Quack game network. In this figure below there are three hypothetical users: Lilly, Brian, and Zack are students; where Mr. $\mathrm{T}$, and Mrs. B, would be teachers and admins of the system.

In conclusion, second grade is a critical year in developing a child's English comprehension. Our prototype, Quack, provides a fun and fresh new way for youngsters to get involved in reading. Although there are similar offerings, Quack's unique utilization of technologies described in this paper, along with its free-to-use mission will hopefully make it a powerful tool for educating youth on English comprehension.

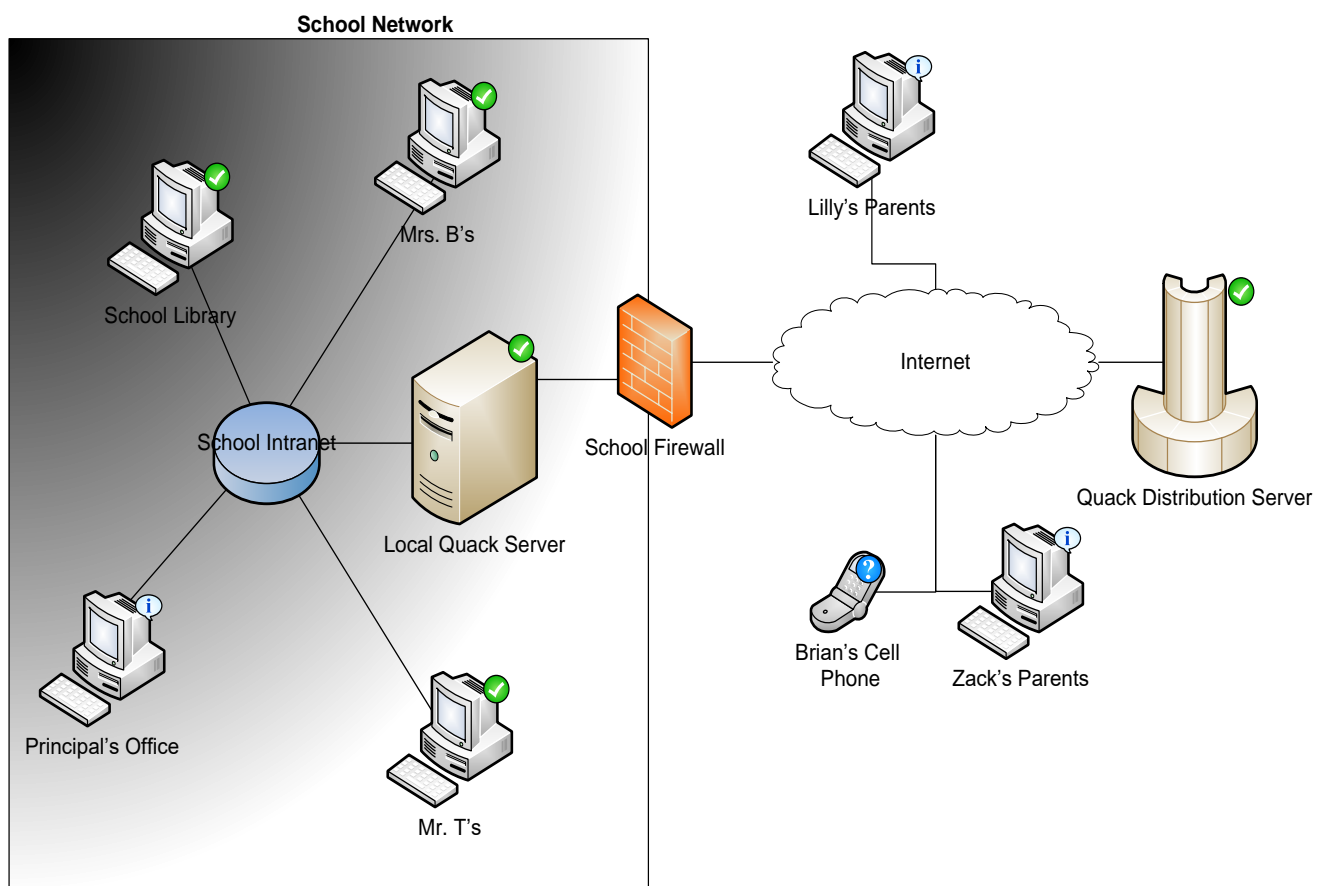

Figure 8: Quack network topology. Green checks indicate minimum topology requirements. Informational bubbles indicate passive users. Question marks indicate platforms that are not supported currently. 


\section{References}

[1] Snow, C., Burns, S., Griffin, P., Preventing Reading Difficulties in Young Children, pg. 247 250

[2] Murray, J., Vanryper, W., Encyclopedia of Graphics File Formats, pg. 17, pg. 187, and pg. 350 370

[3] Young, M., Windows Animation Programming with C++, pg 150 - 154

[4] Angel, E. Interactive Computer Graphics: A top-down approach with OpenGL, pg 10, pg 13, pg $20-24$

[5] Kovach, P., The awesome power of Direct3D/DirectX, pg 54

[6] Greene, J., Stellman, A., Applied Software Project Management, pg 20

[7] ABC Teach, http://www.abcteach.com/directory/reading_comprehension/grades 24/fictional/, Accessed July $11^{\text {th }}, 2018$

[8] Hunt, K., http://www.kendallhunt.com/uploads/2/MTB_Gr2_LitList.pdf, Accessed March 11 ${ }^{\text {th }}$, 2018

[9] Cicerchia, M. https://www.readandspell.com/us/how-to-improve-spelling, Accessed October $24^{\text {th }}$, 2018

[10] A.C. Nielsen Co., http://www.csun.edu/science/health/docs/tv\&health.html, Accessed March $13^{\text {th }}, 2018$

[11] Multiple Authors, Wikipedia, http://en.wikipedia.org/wiki/Sesame_Street, Accessed October 13th, 2018

[12] Szafir, D. and Mutlu, B. Pay attention!: designing adaptive agents that monitor and improve user engagement. In Proceedings of the SIGCHI Conference on Human Factors in Computing Systems (CHI '12). pg 11-20 